\title{
Molecular Dynamics Calculations
}

The development of thermodynamics and statistical mechanics is very important in the history of physics, and it underlines the difficulty in dealing with systems involving many bodies, even if those bodies are identical. Macroscopic systems of atoms typically contain so many particles that it would be virtually impossible to follow the behavior of all of the particles involved. Therefore, the behavior of a complete system can only be described or predicted in statistical ways.

Under a grant to the NASA Lewis Research Center, scientists at the Case Western Reserve University have been examining the use of modern computing techniques that may be able to investigate and find the behavior of complete systems that have a large number of particles by tracking each particle individually. This is the study of molecular dynamics. In contrast to Monte Carlo techniques, which incorporate uncertainty from the outset, molecular dynamics calculations are fully deterministic. Although it is still impossible to track, even on high-speed computers, each particle in a system of a trillion trillion particles, it has been found that such systems can be well simulated by calculating the trajectories of a few thousand particles. Modern computers and efficient computing strategies have been used to calculate the behavior of a few physical systems and are now being employed to study important problems such as supersonic flows in the laboratory and in space.
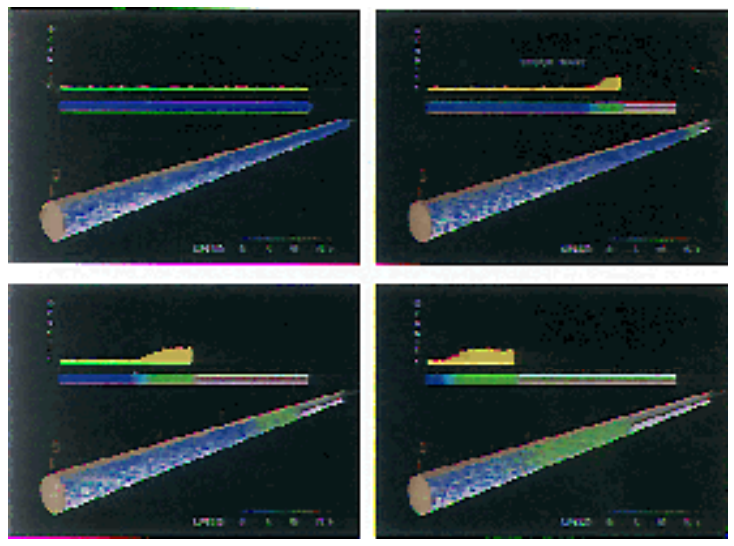

Simulations of shock development.

In particular, an animated video (available in mpeg format--4.4 MB) was produced by Dr. M.J. Woo, now a National Research Council fellow at Lewis, and the G-VIS laboratory at Lewis. This video shows the behavior of supersonic shocks produced by pistons in enclosed cylinders by following exactly the behavior of thousands of particles. The major assumptions made were that the particles involved were hard spheres and that all collisions with the walls and with other particles were fully elastic. The animated video was voted one of two winning videos in a competition held at the meeting of the American Physical Society's Division of Fluid Dynamics, held in Atlanta, Georgia, in November 1994. Of great interest was the result that in every shock there were a few high-speed precursor particles racing ahead of the shock, carrying information about its impending arrival. 


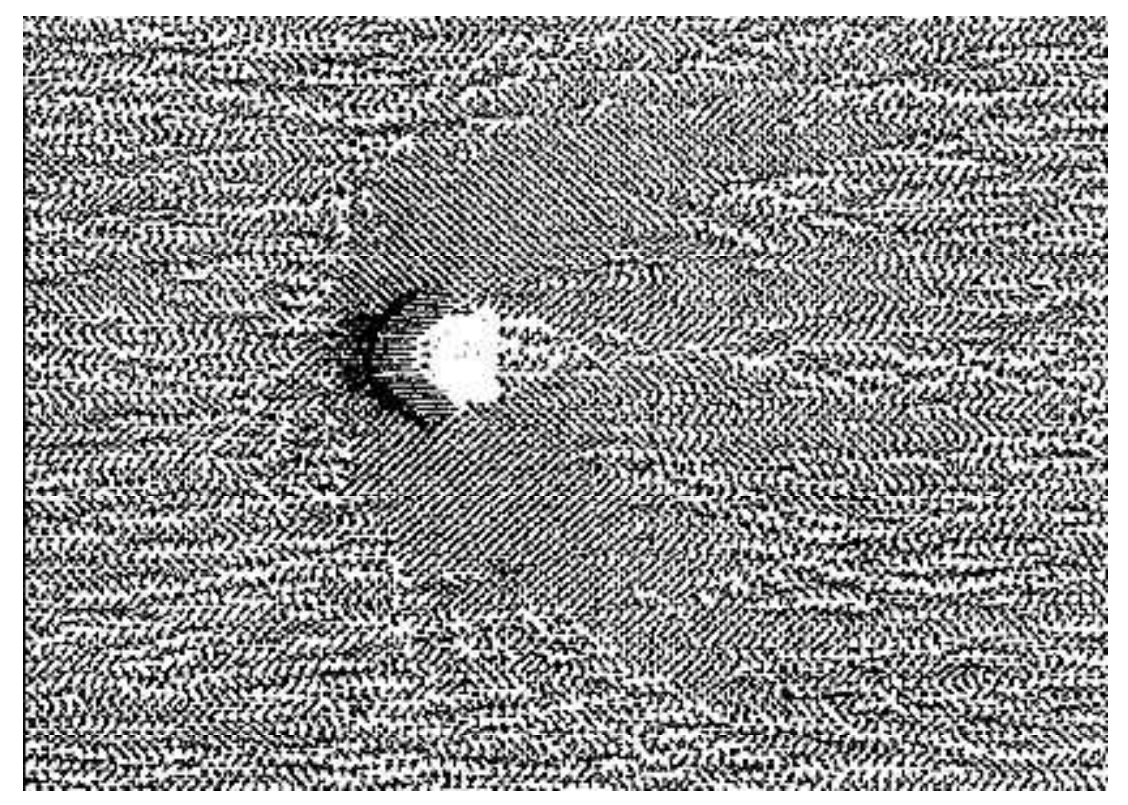

Flow field around the truss structure of the international space station.

Most recently, Dr. Woo has been applying molecular dynamics techniques to the problem of determining the drag produced by the space station truss structure as it flies through the thin residual atmosphere of low-Earth orbit. This problem is made difficult by the complex structure of the truss and by the extreme supersonic nature of the flow. A fully filled section of the truss has already been examined, and drag predictions have been made. Molecular dynamics techniques promise to make realistic drag calculations possible even for very complex partially filled truss segments flying at arbitrary angles. 